\title{
An Algebraic Proof of Quillen's Resolution Theorem for $K_{1}$
}

\author{
Ben Whale*t
}

November 1, 2018

\begin{abstract}
In his 1973 paper [4] Quillen proved a resolution theorem for the $K$-Theory of an exact category; his proof was homotopic in nature. By using the main result of Nenashev's paper [3], we are able to give an algebraic proof of Quillen's Resolution Theorem for $K_{1}$ of an exact category. This represents an advance towards the goal of giving an essentially algebraic subject an algebraic foundation.

Mathematics Subject Classification: 18F25, 19B99

Keywords: Resolution Theorem, $K$-Theory, Exact Category
\end{abstract}

\section{Introduction}

This paper presents an algebraic proof of Quillen's Resolution Theorem of $K_{1}$ of an exact category. The original proof of Quillen's Resolution Theorem, [4] uses homotopic techniques. This research was done with the eventual aim of giving algebraic proofs of most of the major homotopic results in the area of $K$-Theory of exact categories. This would provide a new, and hopefully insightful way, to work in the field.

The paper is divided into three sections. The first reviews the results necessary for this work from Nenashev's three papers, $[1,2]$ and $[3]$ as well as making an important observation about the proof of his main result. The second section presents Quillen's Resolution Theorem and gives the algebraic proof for the case of $K_{1}$ of an exact category. We leave the proof of a lemma to the third and last section as it is long, computational and distracts from the main result.

\footnotetext{
*ben.whale@anu.edu.au

${ }^{\dagger}$ Centre for Gravitational Physics, Department of Physics, Faculty of Science, The Australian National University, Canberra, ACT 0200, Australia
} 
This result was produced as the research component of a $\mathrm{BSc}$ (Hons) under the supervision of Amnon Neeman. The research was done at the Mathematical Sciences Institute of the ANU. Funding was provided by the ANU in the form of an ANU Honours Scholarship. Thank you to Amnon for his suggestions and support, with which this research would not have been completed.

\section{Nenashev and $K_{1}$ of an Exact Category}

Between 1996 and 1998 Nenashev published three papers [1, 2] and [3]. He showed how it was possible to construct a group $D(\mathcal{M})$ from an exact category $\mathcal{M}$ such that there was an isomorphism $D(\mathcal{M}) \stackrel{m}{\longrightarrow} K_{1}(\mathcal{M})$. We begin by giving an overview of the material that we shall need from these papers.

\subsection{Definitions}

1.1.1 Definition. Let $\mathcal{M}$ be an exact category. A double short exact sequence in $\mathcal{M}$, is two short exact sequences on the same objects, $a^{\prime}, a, a^{\prime \prime} \in \mathcal{M}$. That is, a double short exact sequence,

$$
0 \longrightarrow a^{\prime} \stackrel{f_{i}}{\longrightarrow} a \stackrel{g_{i}}{\longrightarrow} a^{\prime \prime} \longrightarrow 0
$$

where $i=1,2$, is really two short exact sequences,

$$
0 \longrightarrow a^{\prime} \stackrel{f_{1}}{\longrightarrow} a \stackrel{g_{1}}{\longrightarrow} a^{\prime \prime} \longrightarrow 0
$$

and

$$
0 \longrightarrow a^{\prime} \stackrel{f_{2}}{\longrightarrow} a \stackrel{g_{2}}{\longrightarrow} a^{\prime \prime} \longrightarrow 0 \text {. }
$$


1.1.2 Definition. A $3 \times 3$ commutative diagram is a diagram

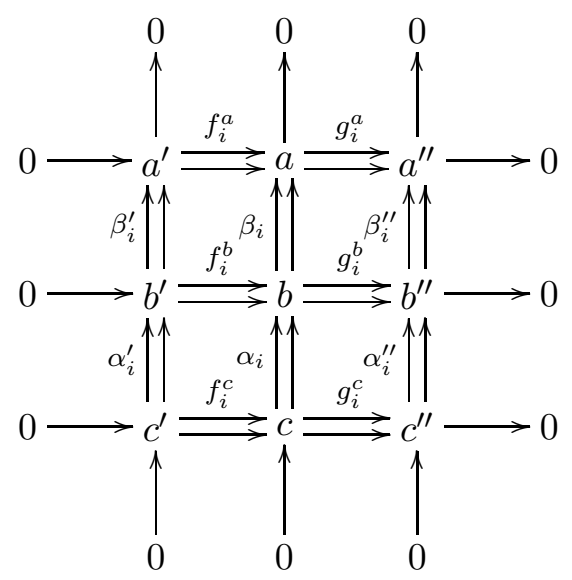

where each row and column is a double short exact sequence such that morphisms of the same subscript commute. Where no subscript is given we shall assume that right maps commute with top maps and left maps with bottom maps. When writing a $3 \times 3$ commutative diagram we shall leave out the 0 objects.

1.1.3 Definition. Given an exact category $\mathcal{M}$, let $D(\mathcal{M})$ be the group with generators $\langle d\rangle$ for all double short exact sequences $d$ in $\mathcal{M}$, and with the relations,

1. Given $<d>=<0 \longrightarrow a^{\prime} \stackrel{f_{i}}{\longrightarrow} a \stackrel{g_{i}}{\longrightarrow} a^{\prime \prime} \longrightarrow 0>$ if $f_{1}=f_{2}$ and $g_{1}=g_{2}$ then $<d>=0$.

2. If there exists a $3 \times 3$ commutative diagram,

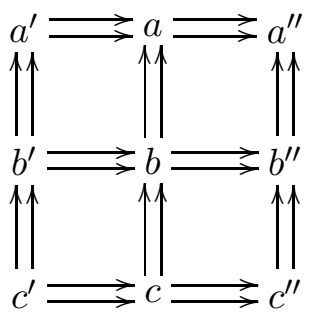


where,

$$
\begin{aligned}
& <H_{T}>=<0 \longrightarrow a^{\prime} \longrightarrow a \longrightarrow a^{\prime \prime} \longrightarrow 0> \\
& <H_{M}>=<0 \longrightarrow b^{\prime} \longrightarrow b \longrightarrow b^{\prime \prime} \longrightarrow 0> \\
& <H_{B}>=<0 \longrightarrow c^{\prime} \longrightarrow c \longrightarrow c^{\prime \prime} \longrightarrow 0> \\
& <V_{L}>=<0 \longrightarrow c^{\prime} \longrightarrow b^{\prime} \longrightarrow a^{\prime} \longrightarrow 0> \\
& <V_{M}>=<0 \longrightarrow c \Longrightarrow b \Longrightarrow a \longrightarrow 0> \\
& <V_{R}>=<0 \longrightarrow c^{\prime \prime} \longrightarrow b^{\prime \prime} \longrightarrow a^{\prime \prime} \longrightarrow 0>
\end{aligned}
$$

then we have the relation,

$$
<H_{T}>-<H_{M}>+<H_{B}>=<V_{L}>-<V_{M}>+<V_{R}>
$$

in $D(\mathcal{M})$.

\section{$1.2 \quad$ A Result about a Double Short Exact Sequence in $D(\mathcal{M})$}

This result was first proved by Nenashev in [3]. We will use it implicitly throughout the rest of this paper.

1.2.1 Lemma. Consider the double short exact sequence

$$
d=0 \longrightarrow a \underset{i_{l}}{\stackrel{i_{r}}{\longrightarrow}} a \oplus a \underset{p_{r}}{\stackrel{-p_{l}}{\longrightarrow}} a \longrightarrow 0
$$

then $<d>=0$ in $D(\mathcal{M})$.

\subsection{Nenashev's Isomorphism}

This theorem is the foundation for our proof of Quillen's Resolution Theorem as it allows us to work with the elements of $K_{1}$ algebraically.

1.3.1 Theorem. For any exact category $\mathcal{M}$, there exists an isomorphism

$$
m: D(\mathcal{M}) \longrightarrow K_{1}(\mathcal{M})
$$

Proof. Refer to the papers $[1,2]$ and [3]. 


\subsection{The Generating Relations of $D(\mathcal{M})$}

In [3] Nenashev constructs an inverse to the group homomorphism $m$. When he does this he only uses a few $3 \times 3$ commutative diagrams, giving certain relations. We can conclude that every relation in $D(\mathcal{M})$ is generated by the few $3 \times 3$ commutative diagrams that he used. After some careful observation the reader will find that each of relations in [3] are given by one of the three following diagrams.
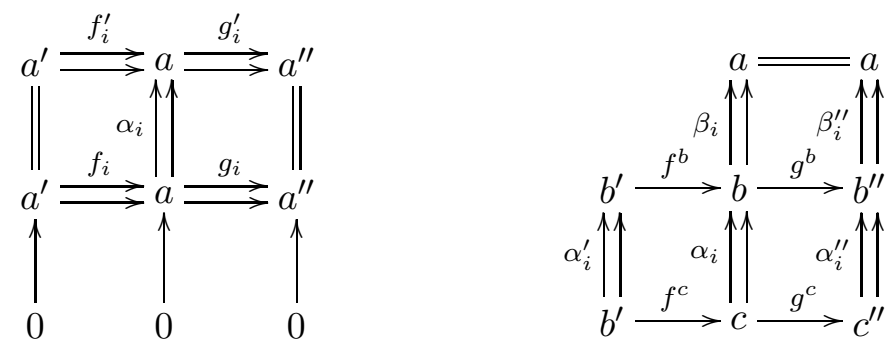

and

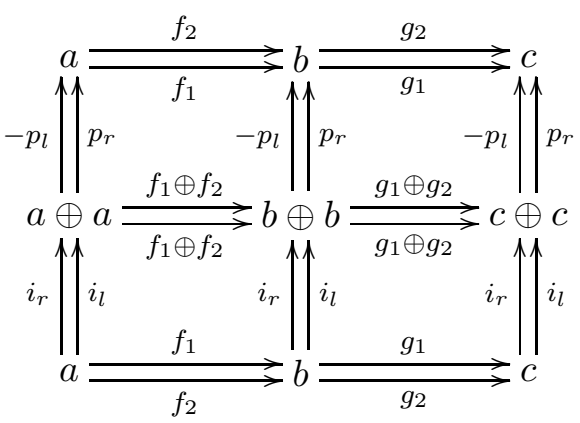

This observation shall be important in our proof of the Resolution Theorem.

\section{The Proof of Quillen's Resolution Theorem for $K_{1}$ using Nenashev's Isomorphism}

We shall prove Quillen's Resolution Theorem for $K_{1}$ of an exact category using the algebraic 'description' of $K_{1}$ given by theorem 1.3.1. Specifically, we shall prove the following theorem:

2.0.1 Theorem (Quillen's Resolution Theorem for $K_{1}$ ). Let $\mathcal{M}$ be an exact category and $\mathcal{F}$ a full subcategory of $\mathcal{M}$ such that for all short exact sequences

$$
0 \longrightarrow a^{\prime} \longrightarrow a \longrightarrow a^{\prime \prime} \longrightarrow 0 \text {, }
$$


1. If $a^{\prime}, a^{\prime \prime} \in \mathcal{F}$ then $a \in \mathcal{F}$,

2. If $a \in \mathcal{F}$ then $a^{\prime} \in \mathcal{F}$,

3. For any $a^{\prime \prime} \in \mathcal{M}$ there exists a short exact sequences, as above, so that $a \in \mathcal{F}$.

Then the inclusion functor $\mathcal{F} \longleftrightarrow \mathcal{M}$ induces an isomorphism

$$
i_{*}: K_{1}(\mathcal{F}) \longrightarrow K_{1}(\mathcal{M}) \text {. }
$$

Quillen's original proof of this theorem may be found in [4].

\section{A Few Remarks}

For the rest of this section we shall assume that $\mathcal{M}$ and $\mathcal{F}$ satisfy the hypotheses of the Resolution Theorem. We shall use results from homological algebra, such as the snake lemma, throughout the rest of this paper. This is justified by the Gabriel-Quillen Embedding Theorems [5, pp 399]. Also, we shall draw a ring around the objects in a $3 \times 3$ commutative diagram if they are known to be in $\mathcal{F}$.

\subsection{Definitions, Some Useful Groups and their Relationships}

2.1.1 Definition. We shall say that a double short exact sequence,

$$
0 \longrightarrow a^{\prime} \longrightarrow a \longrightarrow a^{\prime \prime} \longrightarrow 0
$$

is of type 0 if there are no restrictions on $a^{\prime}$, a and $a^{\prime \prime}$, type 1 if $a^{\prime} \in \mathcal{F}$, type 2 if $a^{\prime}, a \in \mathcal{F}$ and of type 3 if $a^{\prime}, a, a^{\prime \prime} \in \mathcal{F}$.

2.1.2 Definition. For all $j=0,1,2,3$ let $F_{j}$ be the free abelian group with generators $[d]_{j}$, where $d$ is a double short exact sequence of type $j$. Where unambiguous we shall drop the $j$ and write $[d]$.

From definition 2.1.1, we see that we have inclusion homomorphisms between the $F_{j}, F_{3} \stackrel{i_{3}}{\longrightarrow} F_{2} \stackrel{i_{2}}{\longrightarrow} F_{1} \stackrel{i_{1}}{\longrightarrow} F_{0}$.

2.1.3 Definition. For all $j=0,1,2,3$, let $T_{j}$ be the quotient of $F_{j}$ by the relation

$$
\left[0 \longrightarrow a^{\prime} \stackrel{f}{\stackrel{f}{\longrightarrow}} a \stackrel{g}{\underset{g}{\longrightarrow}} a^{\prime \prime} \longrightarrow 0\right]_{j}=0
$$

and the relations given by the $3 \times 3$ commutative diagrams below. 
Case 1. For $j=0$ we include all relations

$$
\left[V_{L}\right]-\left[V_{M}\right]+\left[V_{R}\right]=\left[H_{T}\right]-\left[H_{M}\right]+\left[H_{B}\right]
$$

given by specializations of the $3 \times 3$ commutative diagram

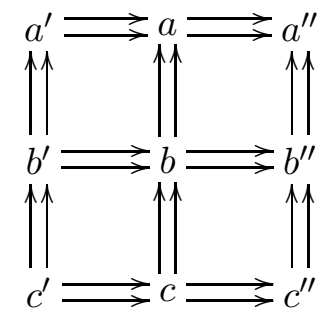

Thus $T_{0} \simeq K_{1}(\mathcal{M})$, by theorem 1.3.1.

Case 2. For $j=1$ we restrict our relations to those given by specializations of the following $3 \times 3$ commutative diagrams; for convenience we write under each $3 \times 3$ commutative diagram the relation that it gives in $T_{1}$.
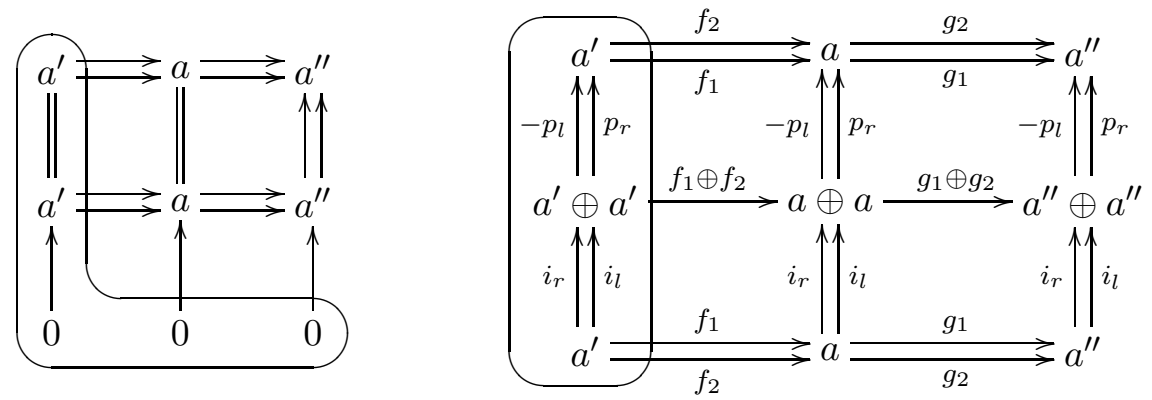

$$
\left[H_{T}\right]-\left[H_{M}\right]=\left[V_{R}\right]
$$

$$
\left[H_{T}\right]+\left[H_{B}\right]=0
$$
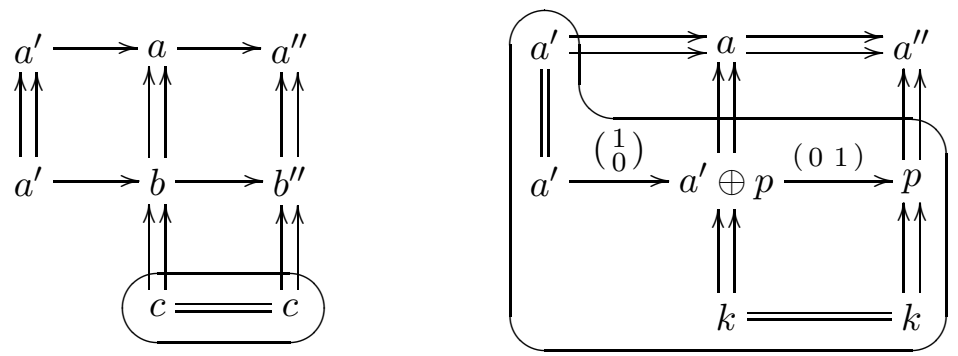

$$
\left[V_{L}\right]-\left[V_{M}\right]+\left[V_{R}\right]=0
$$

$$
\left[H_{T}\right]=\left[V_{R}\right]-\left[V_{M}\right]
$$


Case 3. If $j=2$ we restrict our relations to those given by specializations of the following $3 \times 3$ commutative diagrams; for convenience we write under each $3 \times 3$ commutative diagram the relation that it gives in $T_{2}$.
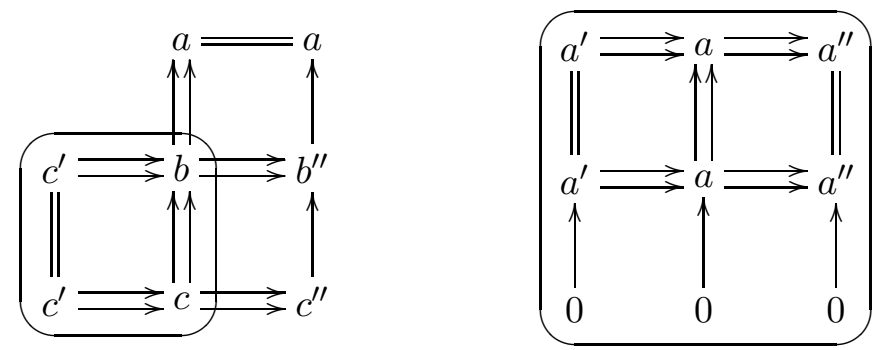

$$
\left[H_{B}\right]-\left[H_{M}\right]=-\left[V_{M}\right] \quad\left[H_{T}\right]-\left[H_{M}\right]=-\left[V_{M}\right]
$$
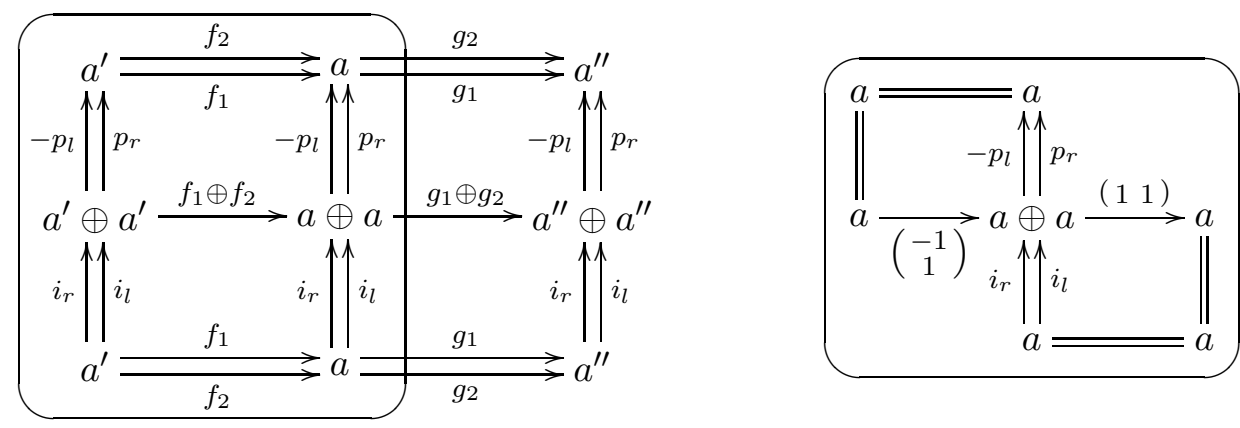

$$
\left[H_{T}\right]+\left[H_{B}\right]=0
$$

$$
\left[V_{M}\right]=0
$$




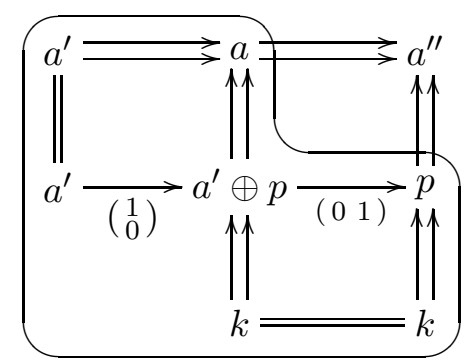

$$
\left[H_{T}\right]=\left[V_{R}\right]-\left[V_{M}\right]
$$

Case 4. If $j=3$ then we allow all relations

$$
\left[V_{L}\right]-\left[V_{M}\right]+\left[V_{R}\right]=\left[H_{T}\right]-\left[H_{M}\right]+\left[H_{B}\right]
$$

given by specializations of the $3 \times 3$ commutative diagram

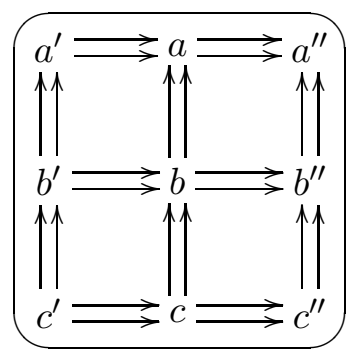

Thus $T_{3} \simeq K_{1}(\mathcal{F})$, by theorem 1.3.1.

In all cases we shall denote the equivalence class of $[d]_{j}$ by $<d>_{j}$. When unambiguous we shall drop the $j$ and simply write $\langle d\rangle$. From the definition of $T_{j}$ it is clear that there exists surjective group homomorphisms $\theta_{j}: F_{j} \longrightarrow T_{j}$, for all $j=0,1,2,3$.

2.1.4 Definition. We shall say that a $3 \times 3$ commutative diagram $D$ is of type $j$ if it is a specialization of one of the diagrams given in the definition of $T_{j}$.

\section{Lemma 1.2.1 revisited}

The $3 \times 3$ commutative diagram used in the proof of lemma 1.2.1 is of type $j$, thus we can conclude that $<0 \longrightarrow a \underset{i_{l}}{\stackrel{i_{r}}{\longrightarrow}} a \oplus a \underset{p_{r}}{\stackrel{-p_{l}}{\longrightarrow}} a \longrightarrow 0>_{j}=0$. 


\subsection{The Group Homomorphisms $\phi_{j}$}

In order to prove the Resolution Theorem we shall construct a group homomorphism that is an inverse to $i_{*}$. To do this we show how we may construct homomorphisms $\phi_{j+1}: F_{j} \longrightarrow F_{j+1}$ that induce functions $\phi_{j+1}^{*}$ between $T_{j}$ and $T_{j+1}$. Before we do this, we present a construction that will allow us to define $\phi_{j+1}$.

\section{The $\phi$-construction}

2.2.1 Construction. For all double short exact sequences $d$

$$
0 \longrightarrow a^{\prime} \stackrel{f_{i}}{\longrightarrow} a \stackrel{g_{i}}{\longrightarrow} a^{\prime \prime} \longrightarrow 0
$$

there exists $p \in \mathcal{F}$ such that we have the commutative triangle

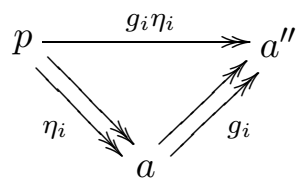

Proof. We construct the pullback of the diagram

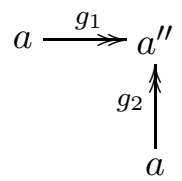

to get the object $a \times_{a^{\prime \prime}} a$. By property (3) of theorem 2.0.1, we can find $p \in \mathcal{F}$ and $\psi: p \longrightarrow a \times_{a^{\prime \prime}} a$, an admissible epimorphism. This gives us the commutative diagram

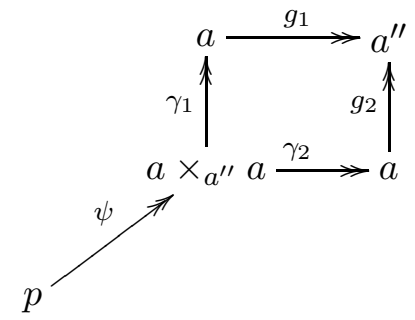

Let $\eta_{i}=\gamma_{i} \psi$. Then we have the commutative triangle

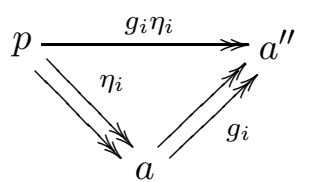


as required.

2.2.2 Construction (The $\phi$-construction). Given a type $j$ double short exact sequence d

$$
0 \longrightarrow a^{\prime} \stackrel{f_{i}}{\longrightarrow} a \stackrel{g_{i}}{\longrightarrow} a^{\prime \prime} \longrightarrow 0
$$

and $p \in \mathcal{F}$ such that we have the commutative triangle

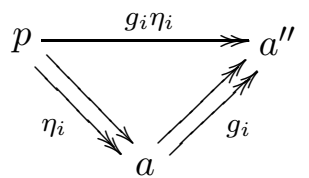

we can construct a type $j+1$ double short exact sequence, $\phi(p, d)$, such that $<d>_{j}=-<\phi(p, d)>_{j}$.

Proof. The commutative triangle allows us to form the $3 \times 3$ commutative diagram

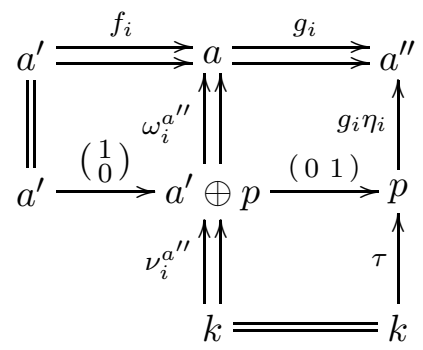

where $\omega_{i}^{a^{\prime \prime}}=\left(f_{i}, \eta_{i}\right)$. By property (2) of theorem 2.0.1 we see that $k \in \mathcal{F}$, thus the double short exact sequence $V_{M}$ is of type $j+1$. We shall denote this double short exact sequence by $\phi(p, d)$. Note that the $3 \times 3$ commutative diagram, above, is of type $j$ thus we have the relation, $\left\langle d>_{j}=-<\right.$ $\phi(p, d)>_{j}$ as required.

We shall often wish to apply construction 2.2.1 to a double short exact sequence $d, 0 \longrightarrow a^{\prime} \stackrel{f_{i}}{\longrightarrow} a \stackrel{g_{i}}{\longrightarrow} a^{\prime \prime} \longrightarrow 0$ and then use the object $p \in$ $\mathcal{F}$, given by construction 2.2 .1 , to apply the $\phi$-construction to $d$. When we do this we shall simply say that we have formed the $\phi$-construction over the morphisms $g_{i}$. 


\section{The Definition of $\phi_{j}$}

As a result of construction 2.2.1, for each double short exact sequence $d$ we may choose $p \in \mathcal{F}$ so that the $\phi$-construction may be applied to $d$ using $p$. We shall denote this $p$ by $p_{d}$.

2.2.3 Definition. Define $\phi_{j+1}: F_{j} \longrightarrow F_{j+1}$ by $\phi_{j+1}\left([d]_{j}\right)=\left[\phi\left(p_{d}, d\right)\right]_{j+1}$ then extend by linearity to the whole group, $F_{j}$.

\subsection{The Proof of The Resolution Theorem}

So far we have constructed a number of groups and group homomorphisms so that we have the diagram,

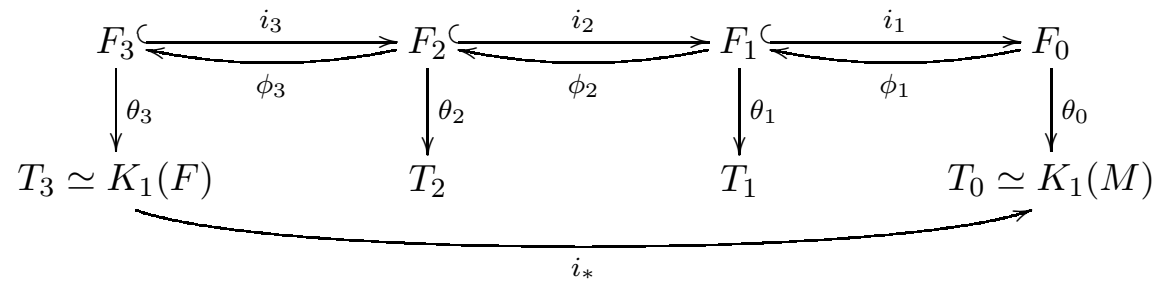

It is possible to see that there are a number of relations between the homomorphisms above. We shall only need two of these relations for our proof.

2.3.1 Lemma. For all $j=1,2,3$, let $\theta_{j}, \phi_{j}, i_{j}$ and $\theta_{0}$ be defined as above. Then we have the two equations,

1. $\theta_{3} \phi_{3} \phi_{2} \phi_{1} i_{1} i_{2} i_{3}=-\theta_{3}$,

2. $\theta_{0} i_{1} i_{2} i_{3} \phi_{3} \phi_{2} \phi_{1}=-\theta_{0}$.

Proof. The proof follows from the fact that $\left\langle\phi\left(p_{d}, d\right)>_{j}=-\left\langle d>_{j}\right.\right.$.

The next lemma is the result that our proof rests on. The proof is long and computational in nature, hence we leave it to the next section.

2.3.2 Key Lemma. The functions $\phi_{j+1}: F_{j} \longrightarrow F_{j+1}$ induce functions $\phi_{j+1}^{*}: T_{j} \longrightarrow T_{j+1}$ such that the diagram

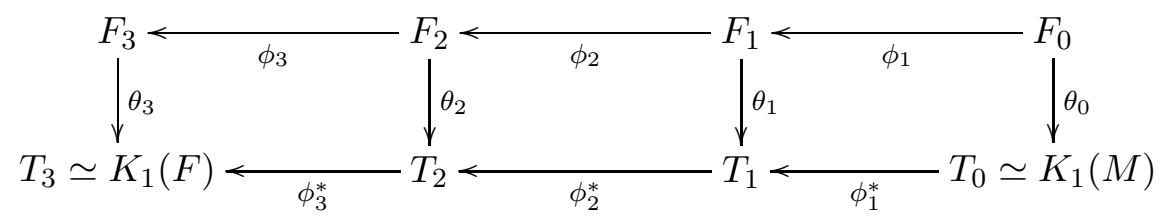

commutes. 
2.3.3 Definition. Define $\varphi: K_{1}(M) \longrightarrow K_{1}(F)$, by $\varphi=\phi_{3}^{*} \phi_{2}^{*} \phi_{1}^{*}$.

We can now prove that the Resolution Theorem follows from Lemma 2.3.2.

Proof of Theorem 2.0.1. We observe that $\varphi \theta_{0}=\theta_{3} \phi_{3} \phi_{2} \phi_{1}$ by the commutativity of diagram (1) and that $i^{*} \theta_{3}=\theta_{0} i_{1} i_{2} i_{3}$ trivially. Hence by lemma 2.3.1) we know that $i^{*} \varphi \theta_{0}=\theta_{0} i_{1} i_{2} i_{3} \phi_{3} \phi_{2} \phi_{1}=-\theta_{0}$ and that $\varphi i^{*} \theta_{3}=$ $\theta_{3} \phi_{3} \phi_{2} \phi_{1} i_{1} i_{2} i_{3}=-\theta_{3}$. Therefore as $\theta_{0}$ and $\theta_{3}$ are both epimorphisms we know that $i^{*} \varphi=\varphi i^{*}=-1_{K_{1}(M)}$. Thus, $-\varphi$ is an inverse to $i^{*}$, and so the inclusion functor $i^{*}: K_{1}(F) \longrightarrow K_{1}(M)$ is an isomorphism, as required.

\section{The Proof of the Key Lemma}

\subsection{Preliminary Results}

In preparation for the results needed to prove the key lemma, we present five constructions.

3.1.1 Construction. Given a specialization of the $3 \times 3$ commutative diagram $D$

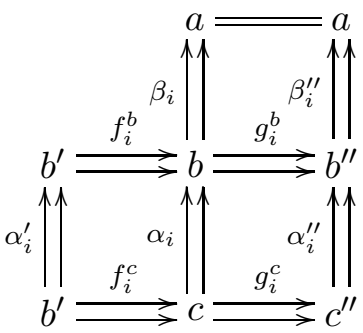

we may construct a specialization of the $3 \times 3$ commutative diagram $D^{\prime}$

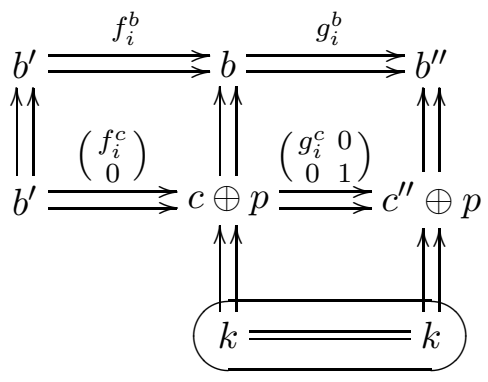

where $V_{M}^{\prime}=\phi\left(p, V_{M}\right)$ and $V_{R}^{\prime}=\phi\left(p, V_{R}\right)$. 
Proof. We take the $\phi$-construction over the two maps $\beta_{i}$, which allows us to construct the two commutative triangles
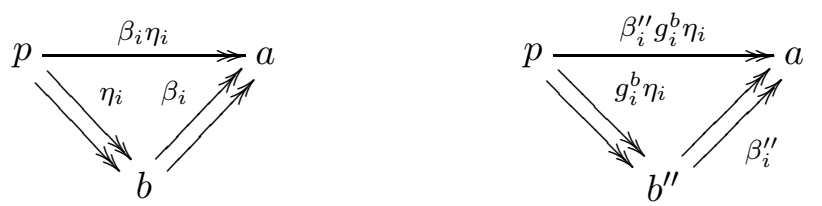

Letting $k=\operatorname{ker}\left(\beta_{i} \eta_{i}\right)$ we find the $3 \times 3$ commutative diagram $D^{\prime}$

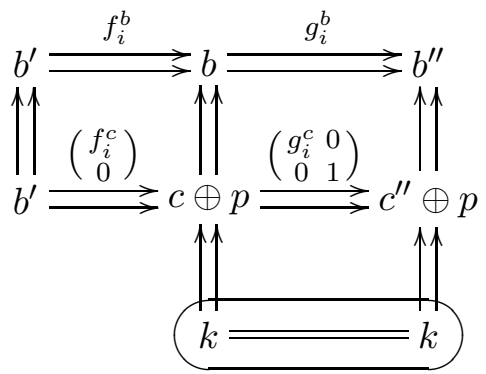

where $V_{M}^{\prime}=\phi\left(p, V_{M}\right)$ and $V_{R}^{\prime}=\phi\left(p, V_{R}\right)$ as required.

3.1.2 Construction. Given a specialization of the $3 \times 3$ commutative diagram $D$

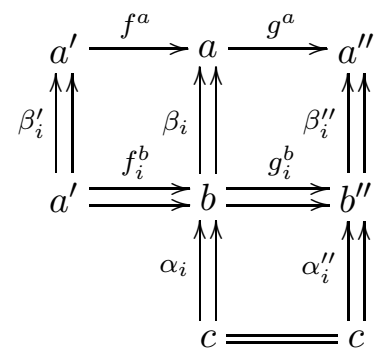

we may construct a specialization of the $3 \times 3$ commutative diagram $D^{\prime}$

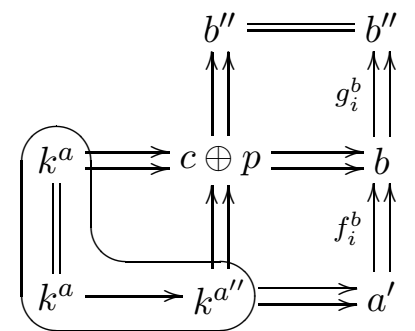

where $H_{M}^{\prime}=\phi\left(p, V_{M}\right)$ and $V_{M}^{\prime}=\phi\left(p, V_{R}\right)$ and if $V_{L}$ is of type $j$ then,

$$
<H_{B}^{\prime}>_{j+1}=<\phi\left(k^{a^{\prime \prime}}, V_{L}\right)>_{j+1} \text {. }
$$


Proof. We form the $\phi$-construction over the maps $\beta_{i}$, to get the two commutative triangles
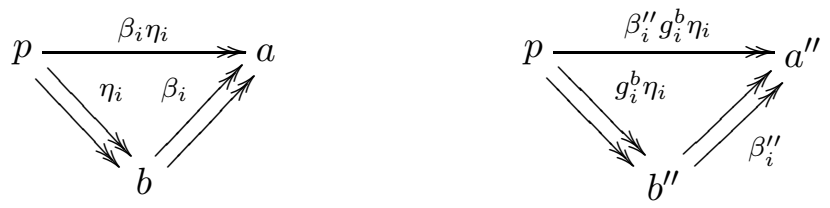

We can construct the $3 \times 3$ commutative diagram

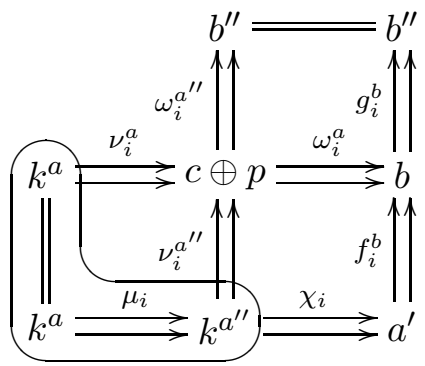

By considering, however, the projections onto $p$ we see that $\mu_{1}=\mu_{2}$. Let $\mu=\mu_{i}$. We now need to compute the map $\chi_{i}$. Note that we have the two commutative diagrams, with short exact rows and columns
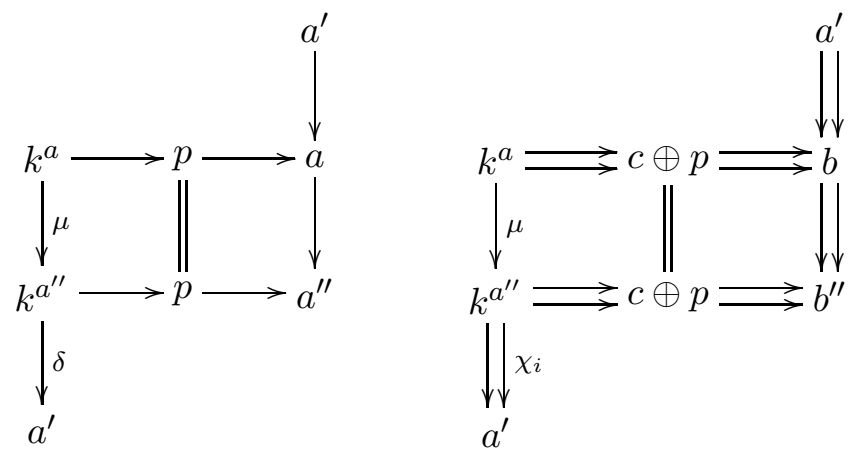

The right diagram maps onto the left diagram, by the obvious maps. These diagrams give us two commutative squares from which it is possible to see that $\chi_{i}=\beta_{i}^{\prime-1} \delta$. Therefore we have the commutative triangle

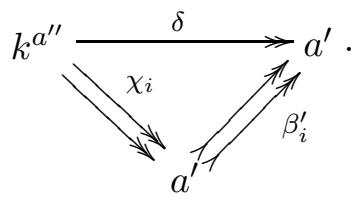


If $V_{L}$ is of type $j$ then the double short exact sequence $\phi\left(k^{a^{\prime \prime}}, V_{L}\right)$ is of type $j+1$ and we have the type $j+13 \times 3$ commutative diagram

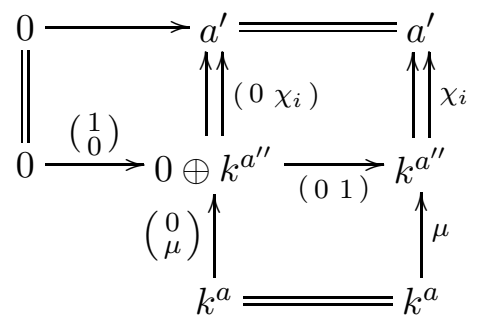

which gives us the relation $<H_{B}^{\prime}>_{j+1}=<\phi\left(k^{a^{\prime \prime}}, V_{L}\right)>_{j+1}$ as required.

3.1.3 Construction. Given a specialization of the $3 \times 3$ commutative diagram $D$

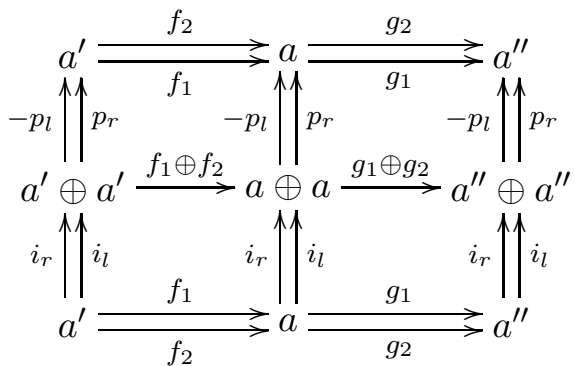

we may construct a specialization of the $3 \times 3$ commutative diagram $D^{\prime}$

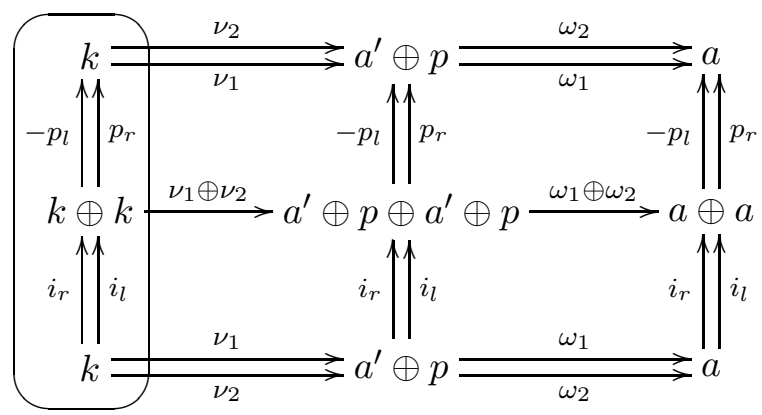

where $H_{T}^{\prime}=\phi\left(p, H_{T}\right)$ and $H_{B}^{\prime}=\phi\left(p, H_{B}\right)$.

Proof. Form the $\phi$-construction over the maps $g_{i}$ to get the commutative diagram

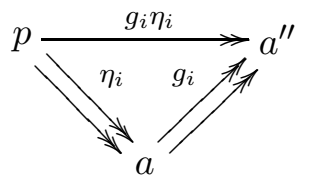


We can then find the $3 \times 3$ commutative diagram

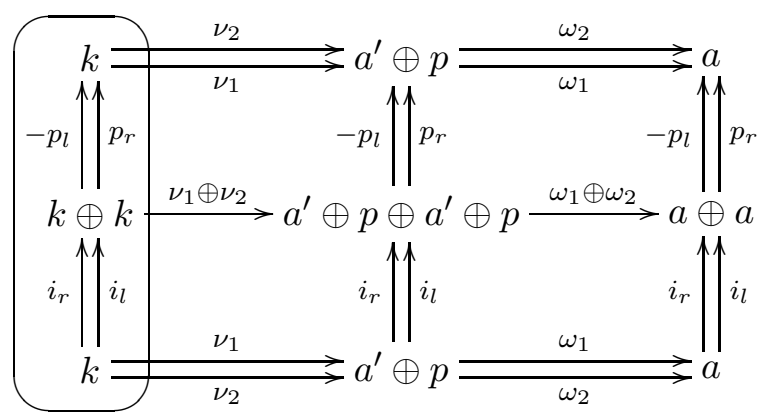

as required.

3.1.4 Construction. Given a $3 \times 3$ commutative diagram which is a specialization of the $3 \times 3$ commutative diagram $D$

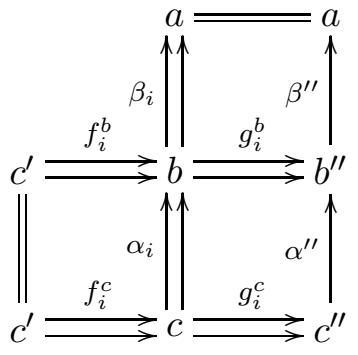

we may construct a specialization of the $3 \times 3$ commutative diagram $D^{\prime}$

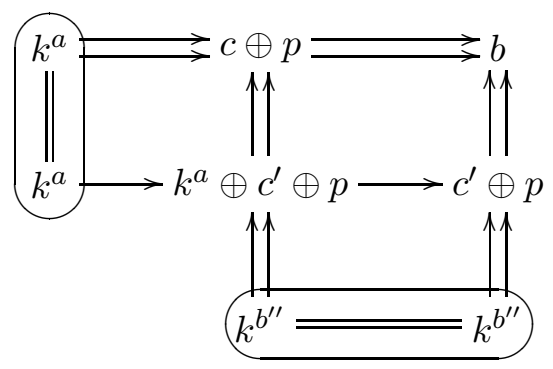

where $H_{T}^{\prime}=\phi\left(p, V_{M}\right), V_{R}^{\prime}=\phi\left(p, H_{M}\right)$ and if $H_{B}$ if of type 2, then

$$
<V_{M}^{\prime}>_{3}=<\phi\left(k^{a}, H_{B}\right)>_{3} .
$$

Proof. We form the $\phi$-construction over the morphisms $g_{i}^{b}$, which allows us 
two construct the two commutative triangles,
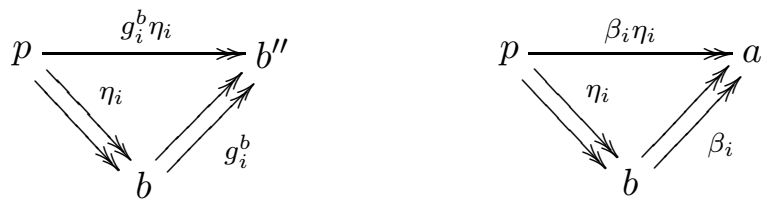

Thus we may also construct the $3 \times 3$ commutative diagram

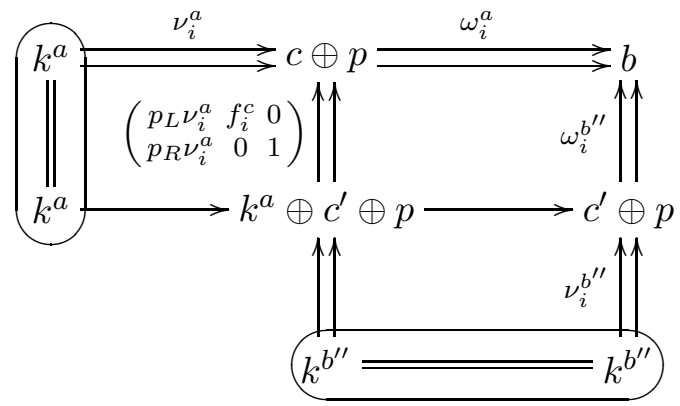

Now suppose that $H_{B}$ is of type 2. We may construct the following commutative diagram

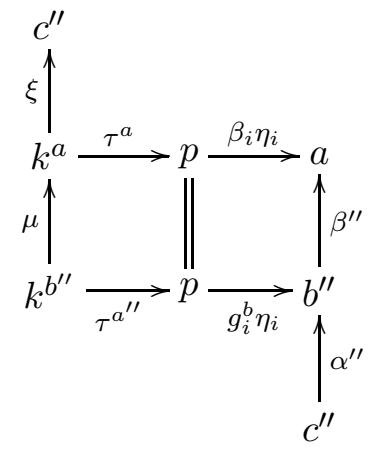

which gives us the relation $\alpha^{\prime \prime} \xi=g_{i}^{b} \eta_{i} \tau^{a}$. Combined with $\omega_{i}^{a} \nu_{i}^{a}=0$ we can show that $\xi=-g_{i}^{c} p_{L} \nu_{i}^{a}$ and hence we know that we have the commutative triangle,

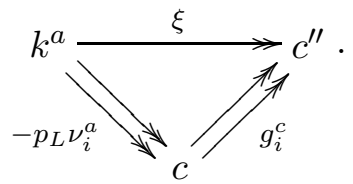

This triangle allows us to construct $\phi\left(k^{a}, H_{M}\right)$ and thus the two type 3 
$3 \times 3$ commutative diagrams
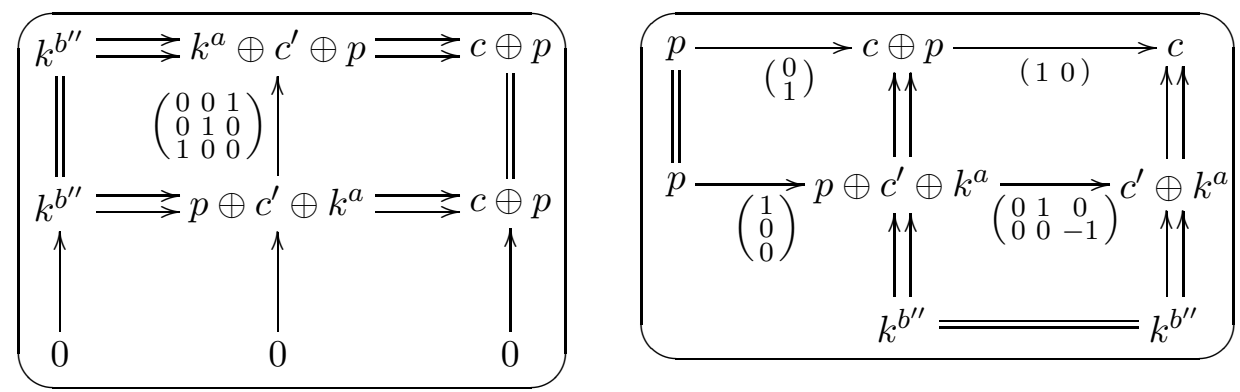

give the relation $<V_{M}^{\prime}>_{3}=<\phi\left(k^{a}, H_{B}\right)>_{3}$ as required.

3.1.5 Construction. Given a specialization of the $3 \times 3$ commutative diagram $D$

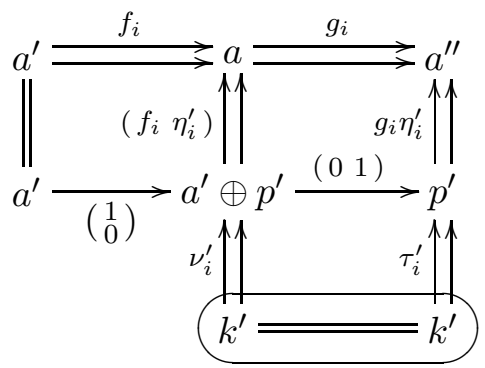

we may construct a specialization of the $3 \times 3$ commutative diagram $D^{\prime}$

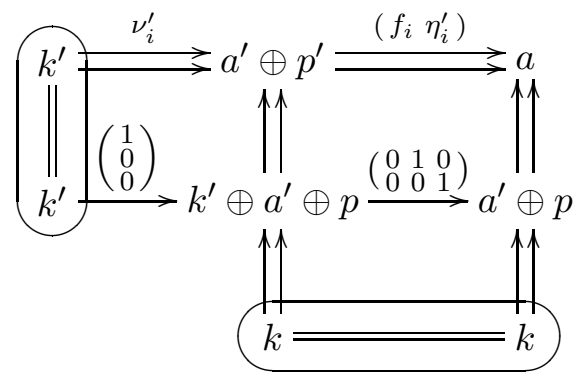

where $V_{R}^{\prime}=\phi\left(p, H_{T}\right)$ and if $D$ is of type $j$ then

$$
<V_{M}^{\prime}>_{j+1}=<\phi\left(p, V_{R}\right)>_{j+1} .
$$

Proof. Form the $\phi$-construction over the maps $g_{i} \eta_{i}^{\prime}$ to get the two commu- 
tative triangles,
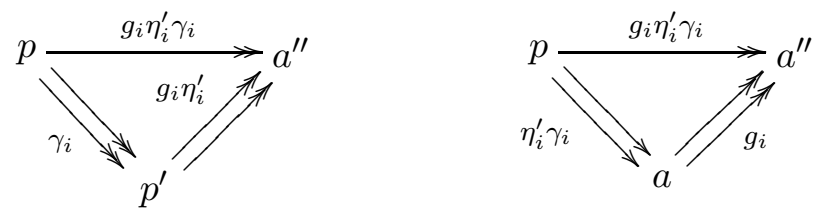

We may construct the type $j+13 \times 3$ commutative diagram

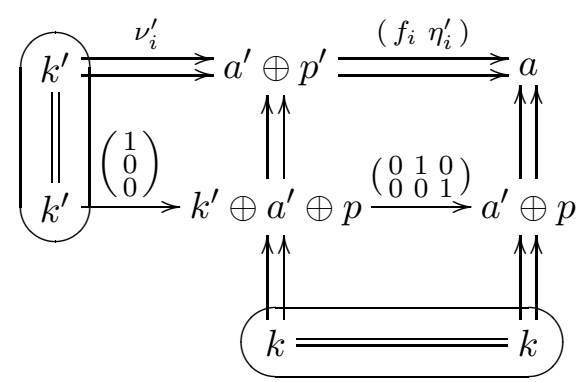

Now, let $D$ be of type $j$. By using the first commutative triangle we can construct $\phi\left(p, V_{R}\right)$, since $\operatorname{ker}\left(\gamma_{i}\right)=\operatorname{ker}\left(g_{i} \eta_{i}^{\prime}\right)=k^{\prime}$. Then we have the two type $j+13 \times 3$ commutative diagrams
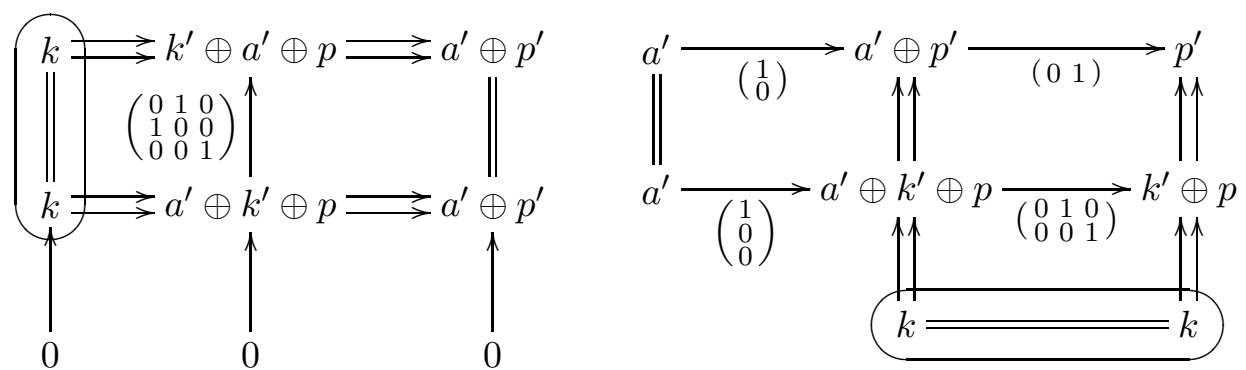

which together give us the relation $<V_{M}^{\prime}>_{j+1}=<\phi\left(p, V_{R}\right)>_{j+1}$, as required.

3.1.6 Construction. Given a specialization of the $3 \times 3$ commutative diagram $D$

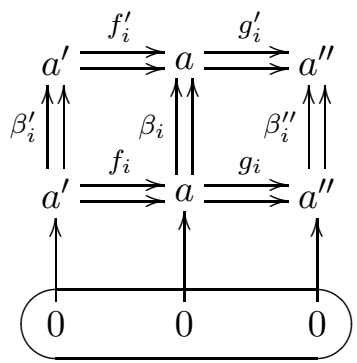


we may construct specializations of the two $3 \times 3$ commutative diagrams $D_{1}$ and $D_{2}$ respectively
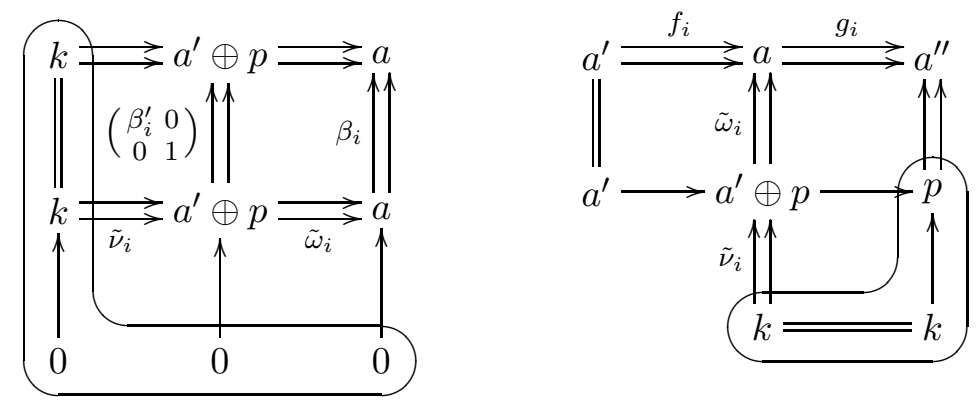

where $H_{T}^{1}=\phi\left(p, H_{T}\right)$ and $V_{R}^{2}=\phi\left(p, V_{R}\right)$.

Proof. We form the $\phi$-construction over the maps $g_{i}^{\prime}$, which gives us the three commutative triangles,
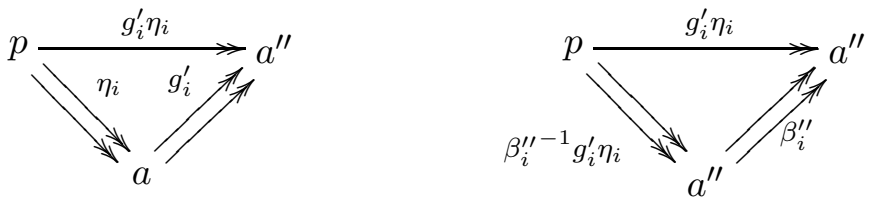

and

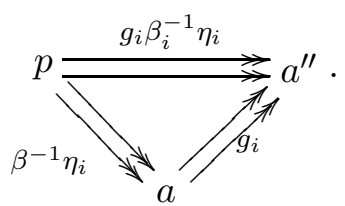

Note that $\operatorname{ker}\left(g_{i} \beta_{i}^{-1} \eta_{i}\right)=\operatorname{ker}\left(g_{i}^{\prime} \eta_{i}\right)$ as $g_{i} \beta_{i}^{-1} \eta_{i}$ differs from $g_{i}^{\prime} \eta_{i}$ by the automorphism $\beta_{i}^{\prime \prime-1}$. Hence from these triangles we form the $3 \times 3$ commutative diagrams
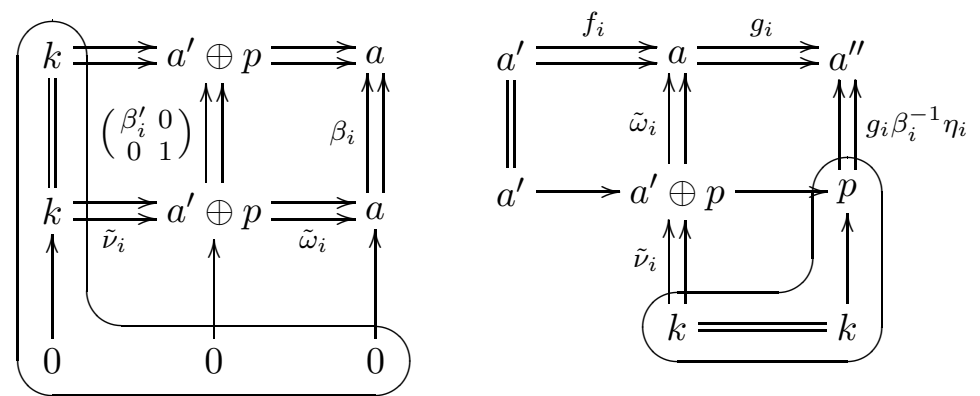

as required. 


\subsection{The Homomorphisms $\theta_{j} \phi_{j}$ are Independent of Choice}

We now give a proof that, for all $j=1,2,3, \theta_{j} \phi_{j}$ is independent of the choice of $p_{d}$. This result is used implicitly throughout the rest of this paper.

3.2.1 Lemma. Let $d=0 \longrightarrow a^{\prime} \stackrel{f_{i}}{\longrightarrow} a \stackrel{g_{i}}{\longrightarrow} a^{\prime \prime} \longrightarrow 0$ be the type $j$ double short exact sequence, and suppose we have the choice of $p$ or $p^{\prime}$ for $p_{d}$. Then $<\phi(p, d)>_{j+1}=<\phi\left(p^{\prime}, d\right)>_{j+1}$.

Proof. Let $p, p^{\prime} \in F$ be such that either are valid choices for $p_{d}$. Then we have two commutative triangles,
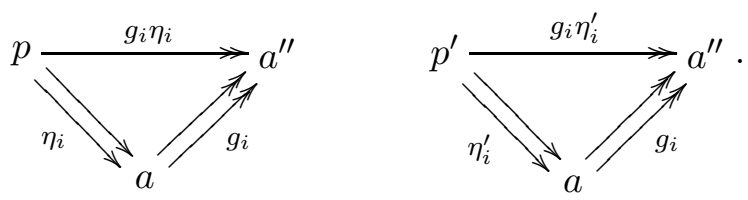

We may form the pullback square

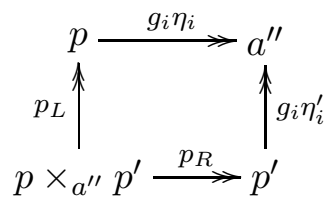

and by property (1) of theorem 2.0.1 we know that $p \times_{a^{\prime \prime}} p^{\prime} \in F$. For ease of notation, let $\tilde{p}=p \times_{a^{\prime \prime}} p^{\prime}$. We will show that $<\phi(p, d)>_{j+1}=<\phi(\tilde{p}, d)>_{j+1}$. Note that $\operatorname{ker}\left(p_{L}\right)=\operatorname{ker}\left(g_{i} \eta_{i}^{\prime}\right)$, so again for ease of notation let $k^{\prime}=\operatorname{ker}\left(p_{L}\right)$.

Case 1 . Suppose that $j=0$. We may construct the $3 \times 3$ commutative diagram

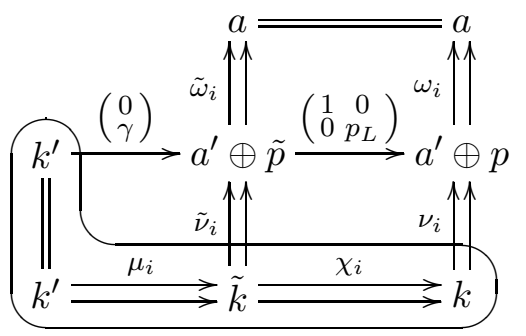

However by considering the projections onto $p$ and $\tilde{p}$ we see that $\chi_{1}=\chi_{2}$ and $\mu_{1}=\mu_{2}$. For consistency of notation, let $\chi=\chi_{i}$ and $\mu=\mu_{i}$. Thus we 
have really constructed the $3 \times 3$ commutative diagram $D$

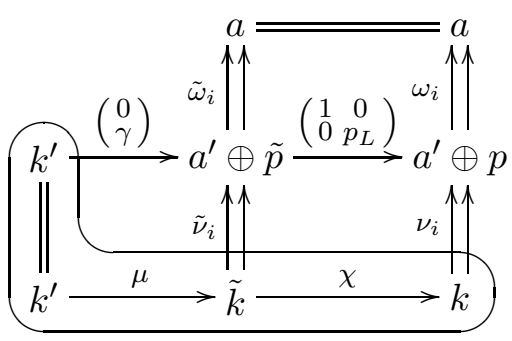

We apply construction 3.1 .1 to $D$ to get the relation $<\phi\left(p_{1}, V_{M}\right)>_{1}=<$ $\phi\left(p_{1}, V_{R}\right)>_{1}$. As $V_{M}$ and $V_{R}$ are both of type 1 we know that $<V_{M}>_{1}=<$ $V_{R}>_{1}$ and hence $<\phi(\tilde{p}, d)>_{1}=<\phi(p, d)>_{1}$ since, by construction, $V_{M}=$ $\phi(\tilde{p}, d)$ and $V_{R}=\phi(p, d)$.

Case 2. Suppose that $j=1,2$. We have the commutative triangle,

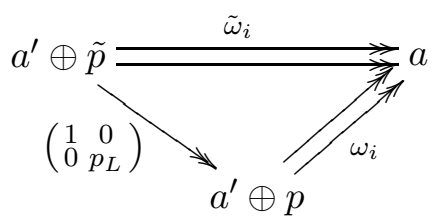

with which we can construct the type $j+13 \times 3$ commutative diagram $D$

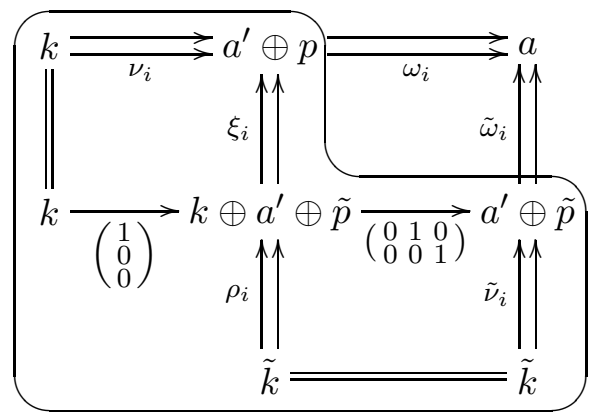

By considering the morphisms $\chi$ and $p_{L}$ we can construct the type $j+1$ $3 \times 3$ commutative diagram

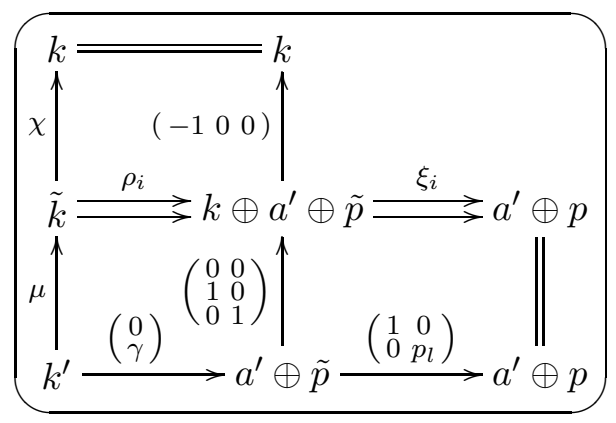


These two $3 \times 3$ commutative diagrams combined give us the relation

$$
<\phi(p, d)>_{j+1}=<\phi(\tilde{p}, d)>_{j+1} .
$$

Hence in either case we see that $<\phi(p, d)>_{j+1}=<\phi(\tilde{p}, d)>_{j+1}$ as was required.

3.2.2 Corollary. For all $j=1,2,3$, the function $\theta_{j} \phi_{j}$ is independent of the choice of $p_{d}$.

Proof. Let $p$ and $p^{\prime}$ be two choices for $p_{d}$. Then the lemma tells us that $<\phi(p, d)>_{j+1}=<\phi\left(p^{\prime}, d\right)>_{j+1}$. Hence we have our result.

Given a double short exact sequence $d$ we shall now denote $<\phi\left(p_{d}, d\right)>_{j}$ by $<\phi(d)>_{j}$, since $<\phi\left(p_{d}, d\right)>_{j}$ is independent of $p_{d}$.

\subsection{The Functions $\phi_{j}$ Induce Group Homomorphisms}

We now need to show that if $\sum_{i=1}^{n} a_{i}<d_{i}>_{j}=0, a_{i} \in \mathbb{Z}$ is a relation then $\sum_{i=1}^{n} a_{i}<\phi\left(d_{i}\right)>_{j+1}=0, a_{i} \in \mathbb{Z}$ is also a relation. To do so we need only check this equation for the generating relations of each group.

\section{The Relations for $T_{0}$}

From the comments and diagrams in subsection 1.4, we have three relations to check.

3.3.1 Lemma. Let $\left\langle H_{T}\right\rangle-\left\langle H_{M}\right\rangle=-\left\langle V_{M}\right\rangle$ be the relation given by the type $03 \times 3$ commutative diagram $D$

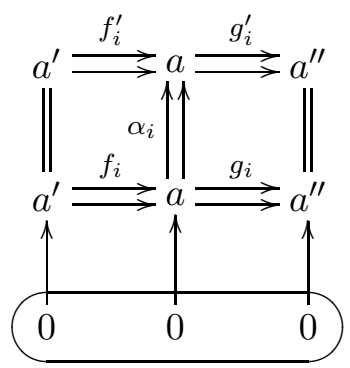

Then $<\phi\left(H_{T}\right)>_{1}-<\phi\left(H_{M}\right)>_{1}=-<\phi\left(V_{M}\right)>_{1}$ is a relation in $T_{1}$.

Proof. We apply construction 3.1.6 to $D$ to get the relation $<\phi\left(H_{T}\right)>_{1}-<$ $\phi\left(H_{M}\right)>_{1}=<V_{M}>_{1}$, but we also know that $<V_{M}>_{1}=-<\phi\left(V_{M}\right)>_{1}$, and so we have the relation $<\phi\left(H_{T}\right)>_{1}-<\phi\left(H_{M}\right)>_{1}=-<\phi\left(V_{M}\right)>_{1}$, as required. 
3.3.2 Lemma. Let $\left\langle V_{L}>-\left\langle V_{M}\right\rangle+\left\langle V_{R}\right\rangle=0\right.$ be the relation given by the type $03 \times 3$ commutative diagram $D$,

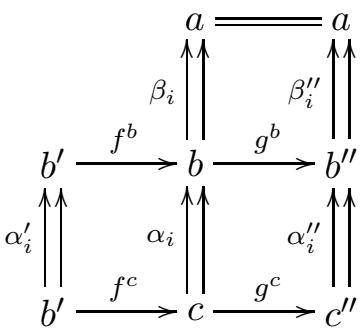

Then $<\phi\left(V_{L}\right)>_{1}-<\phi\left(V_{M}\right)>_{1}+<\phi\left(V_{R}\right)>_{1}=0$ is a relation in $T_{1}$.

Proof. We apply construction 3.1.1 to $D$ and get the relation $<V_{L}^{\prime}>_{1}-<$ $\phi\left(V_{M}\right)>_{1}+<\phi\left(V_{R}\right)>_{1}=0$ and as we have to two type $13 \times 3$ commutative diagrams
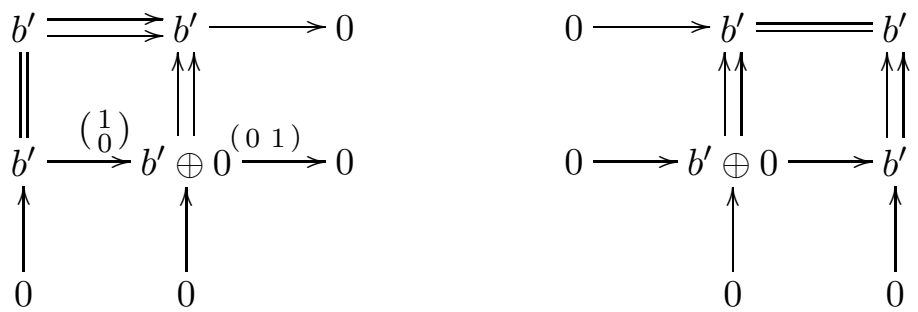

we can see that $<V_{L}^{\prime}>=<\phi\left(V_{L}\right)>$. Therefore we have the relation $<$ $\phi\left(V_{L}\right)>_{1}-<\phi\left(V_{M}\right)>_{1}+<\phi\left(V_{R}\right)>_{1}=0$ as required.

3.3.3 Lemma. Let $\left\langle H_{T}>+<H_{B}>=0\right.$ be the relation given by the type $03 \times 3$ commutative diagram $D$

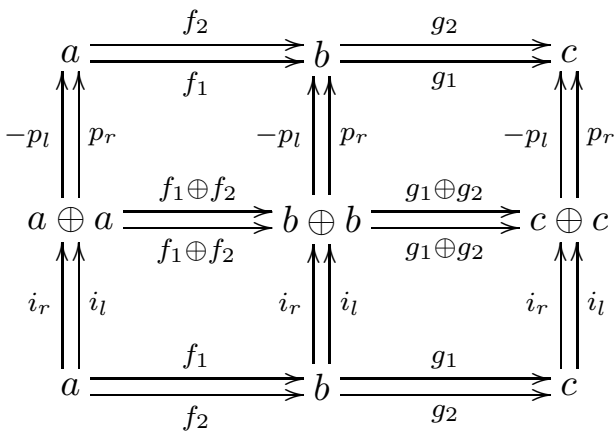

Then $<\phi\left(H_{T}\right)>_{1}+<\phi\left(H_{B}\right)>_{1}=0$ is a relation in $T_{1}$.

Proof. We apply construction 3.1 .3 to $D$ to get the relation $<\phi\left(H_{T}\right)>_{1}$ $+<\phi\left(H_{B}\right)>_{1}=0$ as required. 


\section{The Relations for $T_{1}$}

For $T_{1}$ we need to check the four relations given by the $3 \times 3$ commutative diagrams in definition 2.1.3.

3.3.4 Lemma. Let $\left\langle H_{T}>-<H_{M}>=<V_{R}>\right.$ be the relation given by the type $13 \times 3$ commutative diagram $D$

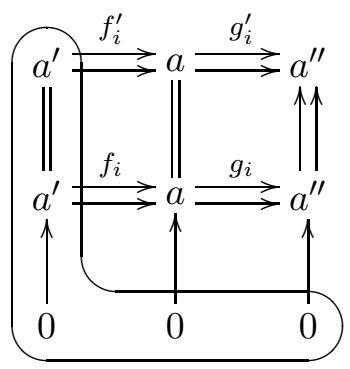

Then $<\phi\left(H_{T}\right)>_{2}-<\phi\left(H_{M}\right)>_{2}=<\phi\left(V_{R}\right)>_{2}$ is a relation in $T_{2}$.

Proof. Apply construction 3.1 .6 to $D$ to get two $3 \times 3$ commutative diagrams $D^{\prime}$ and $D^{\prime \prime}$ respectively
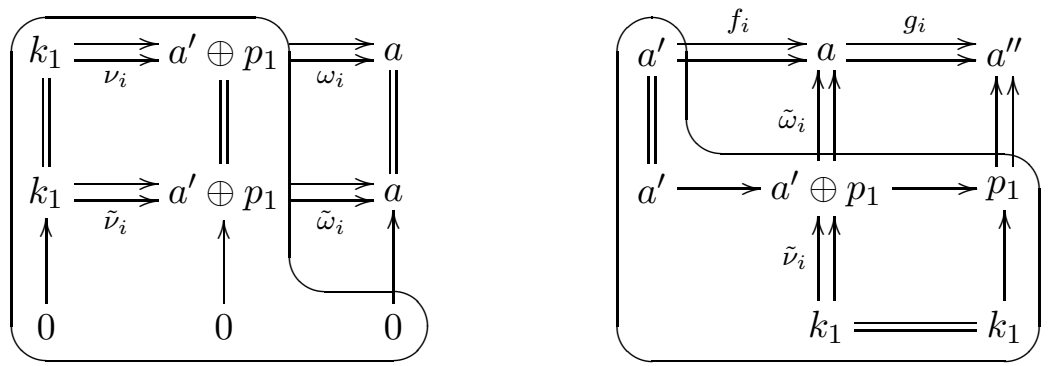

where $H_{T}^{\prime}=\phi\left(H_{T}\right)$ and $V_{R}^{\prime \prime}=\phi\left(V_{R}\right)$. The $3 \times 3$ commutative diagram $D^{\prime}$ tells us that $\omega_{i}=\tilde{\omega}_{i}$ and $\nu_{i}=\tilde{\nu}_{i}$, so when we apply construction 3.1.5 to $D^{\prime \prime}$ we get relation $<\phi\left(H_{T}\right)>_{2}=<\phi\left(H_{M}\right)>_{2}-<\phi^{2}\left(V_{R}\right)>_{2}$. Now, as $\phi\left(V_{R}\right)$ is of type 2 we know that $<\phi\left(V_{R}\right)>_{2}=-<\phi^{2}\left(V_{R}\right)>_{2}$. Hence we get the relation $<\phi\left(H_{T}\right)>_{2}-<\phi\left(H_{M}\right)>_{2}=<\phi\left(V_{R}\right)>_{2}$ as required.

3.3.5 Lemma. Let $\left.\left\langle H_{T}\right\rangle+<H_{B}\right\rangle=0$ be the relation given by the type 
$13 \times 3$ commutative diagram $D$

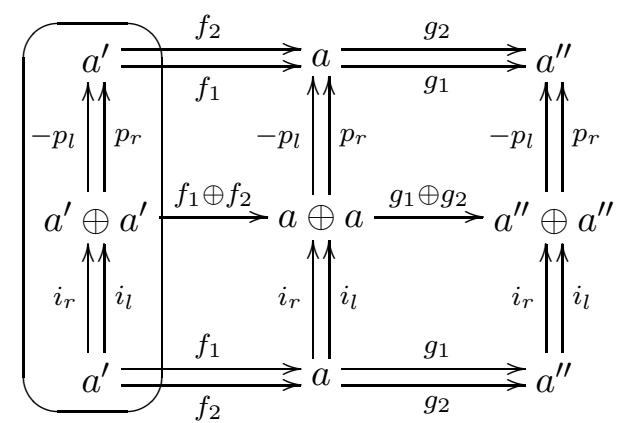

Then $<\phi\left(H_{T}\right)>_{2}+<\phi\left(H_{B}\right)>_{2}=0$ is a relation in $T_{2}$.

Proof. We apply construction 3.1 .3 to $D$ to get the relation $<\phi\left(H_{T}\right)>_{2}$ $+<\phi\left(H_{B}\right)>_{2}=0$ as required.

3.3.6 Lemma. Let $\left\langle V_{L}>-<V_{M}>+<V_{R}>=0\right.$ be the relation given by the type $13 \times 3$ commutative diagram $D$

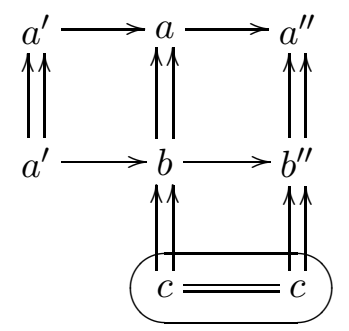

Then the relation $<\phi\left(V_{L}\right)>_{2}-<\phi\left(V_{M}\right)>_{2}+<\phi\left(V_{R}\right)>_{2}=0$ is a relation in $T_{2}$.

Proof. We apply construction 3.1 .2 to $D$ to get the relation $<\phi\left(V_{L}\right)>_{2}$ $-<\phi\left(V_{M}\right)>_{2}+<\phi\left(V_{R}\right)>_{2}=0$ as required.

3.3.7 Lemma. Let $\left\langle H_{T}>=<V_{R}>-<V_{M}>\right.$ be the relation given by the type $13 \times 3$ commutative diagram $D$

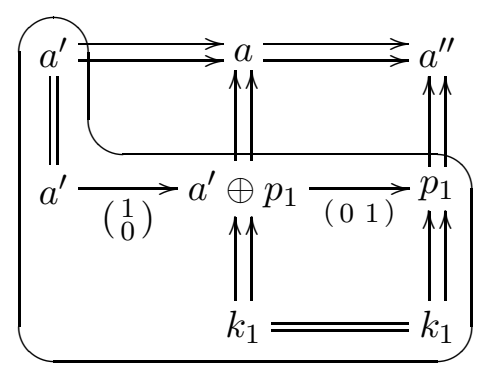


Then $<\phi\left(H_{T}\right)>_{2}=<\phi\left(V_{R}\right)>_{2}-<\phi\left(V_{M}\right)>_{2}$ is a relation in $T_{2}$.

Proof. We apply construction 3.1.5 to $D$ to get the relation $\left\langle V_{M}\right\rangle_{2}=<$ $\phi\left(H_{T}\right)>_{2}-<\phi\left(V_{R}\right)>_{2}$. However, since $V_{M}$ is of type 2 we know that $<V_{M}>_{2}=-<\phi\left(V_{M}\right)>_{2}$. Thus we get the relation $<\phi\left(H_{T}\right)>_{2}=<$ $\phi\left(V_{R}\right)>_{2}-<\phi\left(V_{M}\right)>_{2}$ as required.

\section{The Relations for $T_{2}$}

For $T_{2}$ we need to check the relations given by the $3 \times 3$ commutative diagrams in definition 2.1.3.

3.3.8 Lemma. Let $\left\langle H_{B}>-<H_{M}\right\rangle=-\left\langle V_{M}>\right.$ be the relation given by the type $23 \times 3$ commutative diagram $D$

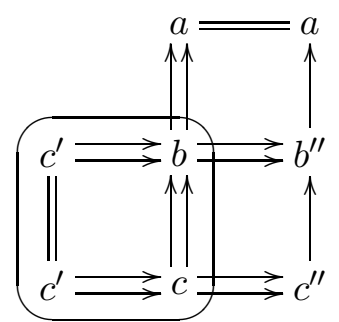

Then $<\phi\left(H_{B}\right)>_{3}-<\phi\left(H_{M}\right)>_{3}=-<\phi\left(V_{M}\right)>_{3}$ is a relation in $T_{3}$.

Proof. We apply construction 3.1.4 to $D$ to get the relation $<\phi\left(V_{M}\right)>_{3}=<$ $\phi\left(H_{M}\right)>_{3}-<\phi\left(H_{B}\right)>_{3}$ as required.

3.3.9 Lemma. Let $\left\langle H_{T}>-<H_{M}>=-\left\langle V_{M}>\right.\right.$ be the relation given by the type $23 \times 3$ commutative diagram $D$

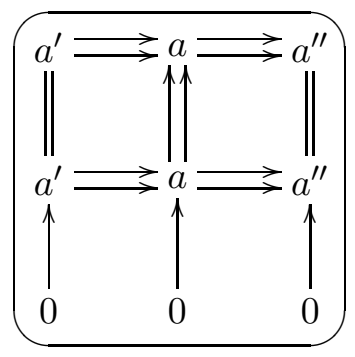

Then $<\phi\left(H_{T}\right)>_{3}-<\phi\left(H_{M}\right)>_{3}=-<\phi\left(V_{M}\right)>_{3}$ is a relation in $T_{3}$.

Proof. Note that $D$ is also of type 3 , hence we have the relation $<H_{T}>_{3}$ $-<H_{M}>_{3}=-<V_{M}>_{3}$. Also, $H_{T}, H_{M}$ and $V_{M}$ are all of type 3 so we know that $<\phi\left(H_{T}\right)>_{3}-<\phi\left(H_{M}\right)>_{3}=-<\phi\left(V_{M}\right)>_{3}$ as required. 
3.3.10 Lemma. Let $\left\langle H_{T}\right\rangle+\left\langle H_{M}\right\rangle=0$ be the relation given by the type $23 \times 3$ commutative diagram $D$

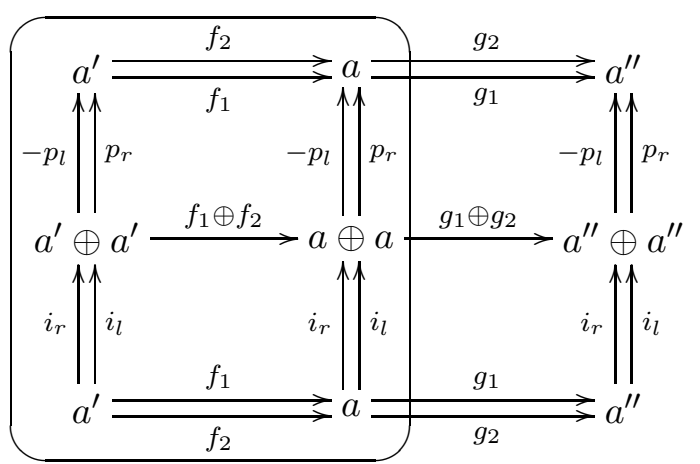

Then $<\phi\left(H_{T}\right)>_{3}+<\phi\left(H_{M}\right)>_{3}=0$ is a relation in $T_{3}$.

Proof. We apply construction 3.1 .3 to $D$ to get the relation $<\phi\left(H_{T}\right)>_{3}$ $+<\phi\left(H_{M}\right)>_{3}=0$ as required.

3.3.11 Lemma. Let $\left\langle V_{M}\right\rangle=0$ be the relation given by the $3 \times 3$ commutative diagram $D$

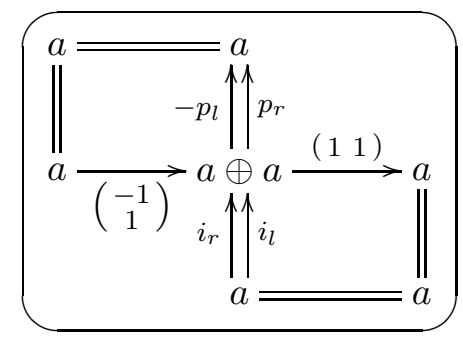

Then $<\phi\left(V_{M}\right)>_{3}=0$ is a relation in $T_{3}$.

Proof. The $3 \times 3$ commutative diagram $D$ is also of type 3 , therefore we have the relation $<V_{M}>_{3}=0$. However, $V_{M}$ is of type 3 thus we know that $<\phi\left(V_{M}\right)>_{3}=0$ as required.

3.3.12 Lemma. Let $\left\langle H_{T}>=<V_{R}>-<V_{M}>\right.$ be the relation given by the type $23 \times 3$ commutative diagram $D$

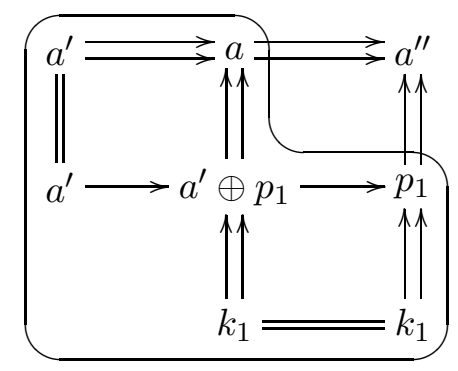


Then $<\phi\left(H_{T}\right)>_{3}=<\phi\left(V_{R}\right)>_{3}-<\phi\left(V_{M}\right)>_{3}$ is a relation in $T_{3}$.

Proof. We apply construction 3.1 .5 to $D$ to get the relation $<V_{M}>_{3}=<$ $\phi\left(H_{T}\right)>_{3}-<\phi\left(V_{R}\right)>_{3}$, but $V_{M}$ is of type 3 and so we know that $<$ $\phi\left(V_{R}\right)>_{3}-<\phi\left(V_{M}\right)>_{3}=<\phi\left(H_{T}\right)>_{3}$ as required.

\section{The Proof of the Key Lemma}

The proof of the key lemma, lemma 2.3.2, follows from the comments at the beginning of this section and from lemmas 3.3.1 to 3.3.12.

\section{References}

[1] A. Nenashev. $K_{1}$ by generators and relations. J. Pure Appl. Algebra, 131(2):195-212, 1998.

[2] Alexander Nenashev. Double short exact sequences produce all elements of Quillen's $K_{1}$. In Algebraic K-theory (Poznań, 1995), volume 199 of Contemp. Math., pages 151-160. Amer. Math. Soc., Providence, RI, 1996.

[3] Alexander Nenashev. Double short exact sequences and $K_{1}$ of an exact category. K-Theory, 14(1):23-41, 1998.

[4] Daniel Quillen. Higher algebraic K-theory. I. In Algebraic K-theory, I: Higher K-theories (Proc. Conf., Battelle Memorial Inst., Seattle, Wash., 1972), pages 85-147. Lecture Notes in Math., Vol. 341. Springer, Berlin, 1973.

[5] R. W. Thomason and T. Trobaugh. Higher algebraic $K$-theory of schemes and of derived categories. In The Grothendieck Festschrift, Vol. III, volume 88 of Progr. Math., pages 247-435. Birkhäuser Boston, Boston, MA, 1990. 